\title{
Bidirectional incompatibility among divergent Wolbachia and incompatibility level differences among closely related Wolbachia in Nasonia
}

\author{
SR Bordenstein ${ }^{1}$ and JH Werren ${ }^{2}$ \\ ${ }^{1}$ Josephine Bay Paul Center for Comparative Molecular Biology and Evolution, The Marine Biological Laboratory, Woods Hole, \\ MA, USA and 'Biology Department, The University of Rochester, Rochester, NY, USA
}

\begin{abstract}
Most insect groups harbor obligate bacterial symbionts from the $\alpha$-proteobacterial genus Wolbachia. These bacteria alter insect reproduction in ways that enhance their cytoplasmic transmission. One of the most common alterations is cytoplasmic incompatibility $(\mathrm{Cl})$ - a post-fertilization modification of the paternal genome that renders embryos inviable or unable to complete diploid development in crosses between infected males and uninfected females or infected females harboring a different strain. The parasitic wasp species complex Nasonia ( $N$. vitripennis, $N$. longicornis and $N$. giraulti) harbor at least six different Wolbachia that cause $\mathrm{Cl}$. Each species have double infections with a representative from both the $A$ and $B$ Wolbachia subgroups. $\mathrm{Cl}$ relationships of the $\mathrm{A}$ and $\mathrm{B}$ Wolbachia of $N$. longicornis with those of $N$. giraulti and $N$. vitripennis are investigated here. We demonstrate that all pairwise crosses between the
\end{abstract}

divergent A strains are bidirectionally incompatible. We were unable to characterize incompatibility between the $B$ Wolbachia, but we establish that the B strain of $N$. longicornis induces no or very weak $\mathrm{Cl}$ in comparison to the closely related B strain in $N$. giraulti that expresses complete $\mathrm{Cl}$. Taken together with previous studies, we show that independent acquisition of divergent A Wolbachia has resulted in three mutually incompatible strains, whereas codivergence of B Wolbachia in N. longicornis and $N$. giraulti is associated with differences in $\mathrm{Cl}$ level. Understanding the diversity and evolution of new incompatibility strains will contribute to a fuller understanding of Wolbachia invasion dynamics and Wolbachia-assisted speciation in certain groups of insects.

Heredity (2007) 99, 278-287; doi:10.1038/sj.hdy.6800994; published online 23 May 2007

Keywords: endosymbiont; cytoplasmic incompatibility; parasitic wasp; postzygotic isolation; Wolbachia

\section{Introduction}

Wolbachia are widespread endosymbiotic bacteria that are found predominantly in the germlines of arthropods and nematodes (Werren, 1997; Stouthamer et al., 1999; Stevens et al., 2001). Their main mode of transmission within species is maternal from ovaries to eggs, but horizontal transmission must also occur between species to account for the wide range of infected hosts. By manipulating arthropod reproduction through male killing, parthenogenesis, feminization and cytoplasmic incompatibility (CI), Wolbachia increase the relative number of infected females (that is, the transmitting sex) in a host population, and thereby spread rapidly within a host species (Caspari and Watson, 1959; Turelli and Hoffmann, 1991; Turelli, 1994; Werren and O'Neill, 1997). These reproductive alterations can also have important implications to basic processes such as sex determination (Rigaud et al., 1997; Werren and Beukeboom, 1998), sexual selection (Jiggins et al., 2000) and speciation (Laven, 1957; Breeuwer and Werren, 1990;

Correspondence: $\operatorname{Dr}$ SR Bordenstein, Josephine Bay Paul Center for Comparative Molecular Biology and Evolution, The Marine Biological Laboratory, 7 MBL Street, Woods Hole, MA 02543, USA.

E-mail: sbordenstein@mbl.edu

Received 28 November 2006; revised 7 March 2007; accepted 19 April 2007; published online 23 May 2007
Bordenstein et al., 2001; Bordenstein, 2003; Jaenike et al., 2006; Koukou et al., 2006). Between arthropod species, horizontal transmission is common on an evolutionary time scale (Werren et al., 1995a; Sintupachee et al., 2006) and has been observed in the laboratory under certain circumstances (Heath et al., 1999; Boyle et al., 1993; Rigaud et al., 2001; Huigens et al., 2004; Frydman et al, 2006).

$\mathrm{CI}$ is the most commonly detected type of Wolbachiainduced reproductive alteration. It is a sperm-egg incompatibility expressed in crosses between an infected male and uninfected female. Although the genetic and biochemical mechanisms of CI are not known, the cytological effects are clear. Sperms that are 'modified' by Wolbachia in the testes show abnormal processing after fertilization of the egg, if the appropriate Wolbachia are not present in the egg to 'rescue' the modification (Werren, 1997). In particular, breakdown of the nuclear envelope of the male pronucleus is delayed (Tram and Sullivan, 2002) and the paternal chromatin undergoes improper condensation during early mitotic divisions (O'Neill and Karr, 1990; Reed and Werren, 1995; Callaini et al., 1997; Tram et al., 2006). As a result, most embryos usually die, but in some haplodiploid species haploidization of the embryo results in male development (Reed and Werren, 1995; Tram et al., 2006). When both male and female are infected by Wolbachia with the same 'modification-rescue' system, the sperm modification is 
rescued in eggs, and compatibility is restored (Werren, 1997). However, if a male and female harbor strains of Wolbachia with different 'modification-rescue' systems, then bidirectional CI results (Perrot-Minnot et al., 1996; Werren, 1997; Charlat et al., 2001). Such strains are referred to as '(in)compatibility types' and have been observed in various insects, including mosquitoes, fruit flies and parasitic wasps (Laven, 1957; Breeuwer and Werren, 1990; O'Neill and Karr, 1990; MontchampMoreau et al., 1991; Perrot-Minnot et al., 1996; James and Ballard, 2000; Bordenstein et al., 2001; Dedeine et al., 2004). Bidirectional CI has attracted considerable attention for its potential role in driving rapid speciation, because gene flow between diverging populations that harbor different Wolbachia incompatibility types, can be reduced or eliminated due to endosymbionts ( $\mathrm{O}^{\prime} \mathrm{Neill}$ and Karr, 1990; Werren, 1998; Bordenstein et al., 2001; Telschow et al., 2002; Bordenstein, 2003). The effect can also select for premating isolation (Telschow et al., 2005a, b).

Among the eight major subgroups of Wolbachia (Lo et al., 2002; Rowley et al., 2004; Bordenstein and Rosengaus, 2005), the A and B groups are most commonly found in insects and diverged approximately 60 million years ago (Werren et al., 1995a). Multiple infections occur at appreciable frequencies throughout a wide range of insect species (Werren et al., 1995a, b; Werren and Windsor, 2000; Jeyaprakash and Hoy, 2000). In the parasitic wasp genus Nasonia, all three species $(N$. vitripennis, N. giraulti and $N$. longicornis) are coinfected by each of the two major insect Wolbachia subdivisions, A and B (Breeuwer et al., 1992; Werren et al., 1995a; Werren and Bartos, 2001; van Opijnen et al., 2005). Nearly all field samples within these three species harbor the double AB infections (Bordenstein et al., 2001). This genus is therefore particularly useful for studying the CI phenomenon as they are prone to acquiring and maintaining genetically distinct Wolbachia. Some isolates of $N$. longicornis are now known to carry two very closely related B Wolbachia strains along with the A Wolbachia strain ( $\mathrm{R}$ Choudury, personal communication). The IV7 isolate used in this study, however, is only infected with one A and one B Wolbachia.

These three wasp species are reproductively isolated in the laboratory owing to Wolbachia-induced bidirectional incompatibility between the different, double AB infections (Breeuwer and Werren, 1990; Bordenstein et al., 2001). CI also produces distinct phenotypes among the Nasonia species: embryonic mortality in $N$. longicornis and $N$. giraulti due to missegregation of the paternal chromosomes, and conversion to male development in $N$. vitripennis due to exclusion of the paternal genome from embryonic development (Bordenstein et al., 2003; Tram et al., 2006). Although there are low levels of conversion (10-20\%) in N. longicornis and N. giraulti and low levels of embryonic mortality in $N$. vitripennis, a genetic analysis showed the major difference underlying the mortality/conversion phenotype is a Nasonia host genetic effect rather than Wolbachia strain differences (Bordenstein et al., 2003).

In this paper, we determine $\mathrm{CI}$ among the single $\mathrm{A}$ and B Wolbachia in Nasonia and how the incompatibility relationships associate with genetic divergence among the Wolbachia strains. The phylogenetic data thus far suggest that five Wolbachia infections entered the Nasonia

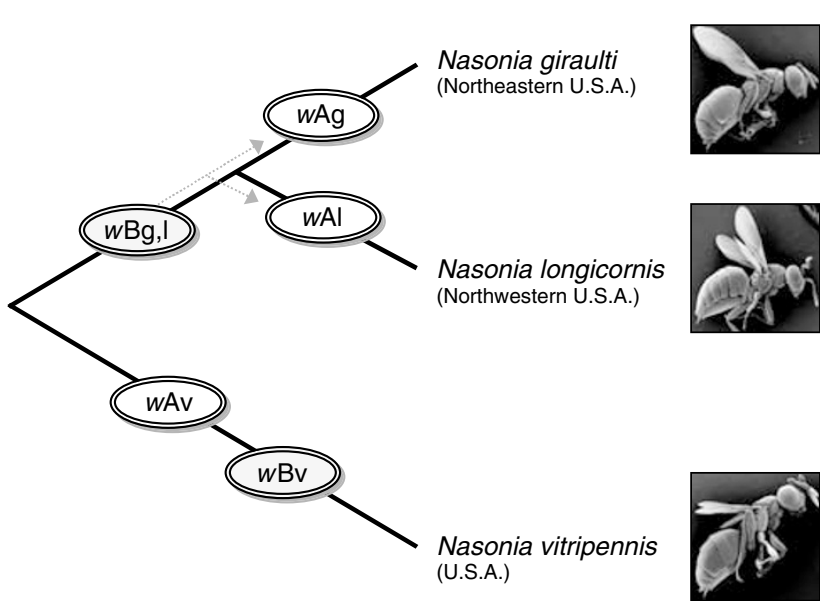

Figure 1 A schematic phylogeny showing the hypothesized origin of A (white circles) and B (gray circles) Wolbachia in Nasonia (redrawn from van Opijnen et al., 2005). All three A and two B Wolbachia strains were independently acquired in the three Nasonia species by horizontal transfer from another insect. The $w \mathrm{Bg}, 1$ infection then likely codiverged with $N$. giraulti and N. longicornis, denoted by the dotted lines with arrowheads. Regional species distributions are noted in parentheses. Scanning electron micrographs of Nasonia males (Copyright Dennis Kunkel Microscopy, Inc.) show the major morphological difference (that is, male wing size) between the three closely related species, with $N$. giraulti, $N$. longicornis and $N$. vitripennis having the largest, intermediate and smallest wing sizes, respectively. An italicized lower case $w$ followed by a capital A or B denotes the subgroup of Wolbachio (for example, $w \mathrm{~A}$ ). The lower case $\mathrm{v}, \mathrm{g}$ or 1 that follows specifies whether the strain is derived from $N$. vitripennis, $N$. giraulti or $N$. longicornis. For example, wAl symbolizes the $N$. longicornis A Wolbachia.

system laterally and one codiverged with its host species (Figure 1). These phylogenetic inferences are based on three lines of evidence. First, each of the three A Wolbachia show more wsp (Wolbachia surface protein gene) nucleotide similarity to strains found in other insects than to those strains infecting other Nasonia species (van Opijnen et al., 2005). For instance, the A infection in $N$. longicornis shows no synonymous divergence to that of Drosophila melanogaster (wMel) and $D$. simulans $(w \mathrm{Au})$, yet it shows 14.44 and $8.86 \%$ synonymous divergence to the $\mathrm{A}$ infection in N. vitripennis and $N$. giraulti, respectively. Further, extrapolated divergence times for the A Wolbachia in these Nasonia species $(9.0$ and 5.5 Mya, respectively) are greater than the estimated time of the most recent common ancestor of the three Nasonia species (Campbell et al., 1993). The group B Wolbachia show a similar trend except for the strains in $N$. giraulti and $N$. longicornis. These B strains show no wsp synonymous divergence, although they are $26.81 \%$ divergent to the $\mathrm{B}$ infection in $N$. vitripennis. These strains presumably codiverged with the ancestor of the B Wolbachia in N. giraulti and N. longicornis, sister species that are estimated to have diverged only a few 100000 years ago (Campbell et al., 1993; van Opijnen et al., 2005). They remain one of only a few documented instances of codivergence of Wolbachia and their insect hosts. The above nucleotide patterns are also observed with additional genes, including $16 \mathrm{~S}$ rDNA and seven proteincoding genes (Breeuwer et al., 1992; Werren et al., 1995a; van Opijnen et al., 2005; Casiraghi et al., 2005; Baldo et al., 2006). Together, the data suggest that the three $A$ 
Wolbachia and two B Wolbachia of Nasonia were independently acquired by horizontal transfer from insects outside the genus. The exception of the B group Wolbachia in $N$. giraulti and $N$. longicornis indicates codivergence with these sister species. Finally, a low level of nucleotide diversity among each infection suggests that all of the Wolbachia were acquired too recently to have had time to accumulate much polymorphism. This scenario is consistent with the proposed origins of the Nasonia Wolbachia.

A major question regarding the evolution of CIinducing Wolbachia is how codivergence of closely related strains or lateral acquisition of divergent strains into the same host species influence the expression and evolution of new CI strains. We address three questions related to this topic: (i) Do the distantly related A infections of each species constitute three distinct incompatibility types? (ii) Do the closely related B infections of $N$. giraulti and $N$. longicornis differ in CI? and (iii) Does the host species genotype influence bidirectional incompatibility between double infections of $N$. giraulti and N. longicornis? These questions have important implications for the origin of new incompatibility types, the rate at which new ones can evolve, and the significance of host-Wolbachia genetic interactions in shaping CI patterns.

\section{Materials and methods}

Nasonia are gregarious parasitoid wasps of fly pupae. An introduction to Nasonia biology can be found in Whiting (1967). They are raised on Sarcophaga bullata (flesh fly) pupae in the laboratory, with constant light and temperature $\left(25^{\circ} \mathrm{C}\right)$. Under these conditions, generation time is 14 days for $N$. vitripennis and 15 days for $N$. giraulti and N. longicornis. These insects have haplodiploid sex determination, so females are diploid and develop from fertilized eggs, whereas males are haploid and develop from unfertilized eggs.

\section{Nomenclature}

Individuals in each Nasonia species are double infected with an A and B Wolbachia strain, comprising at least six strains in the genus. For the purposes of this paper, we will use a shorthand nomenclature system to refer to these strains in the text and figures. An italicized lower case $w$ followed by a capital A or B denotes the subgroup of Wolbachia (for example, wA). Zero in place of this denotes an uninfected host. The lower case $\mathrm{v}, \mathrm{g}$ or 1 that follows specifies whether the strain is derived from $N$. vitripennis, N. giraulti or N. longicornis. And finally, when describing crosses with wasps harboring the Wolbachia of another species (that is, introgression lines), the entire designation is enclosed in brackets, and a capital V, G or $\mathrm{L}$ follows to indicate the host species genetic background. Thus, [wAl]L symbolizes the $N$. longicornis $\mathrm{A}$ Wolbachia in the N. longicornis host genetic background.

\section{Insect strains}

Eight laboratory insect strains were used to test CI when Wolbachia occur in their resident species background. Two N. vitripennis strains were used: [wAv]V is a single A-infected lab strain (named 12.1) and [0v]V is an uninfected strain (named 13.2). Both were derived from a double-infected wild-type strain by spontaneous loss of
Wolbachia after prolonged diapause (Perrot-Minnot et al., 1996). Three $N$. giraulti strains were used: [wAg, $w \mathrm{Bg}] \mathrm{G}$ is double-infected (RV2), [0g]G is uninfected (RV2R) and [wAg]G is single A-infected (16.2 RV2D). The latter two strains were both derived from RV2 through antibiotic treatments in 1996, and diapause treatment in 1998, respectively. Similarly for $N$. longicornis, $[w \mathrm{Al}, w \mathrm{Bl}] \mathrm{L}$ is double-infected (IV7), [01]L is uninfected (IV7R3-1B and [wAl]L is single A-infected (2.1 IV7D). In this species, the latter two strains were derived from IV7 through antibiotic treatment in 2000, and diapause treatment in 1998, respectively. Attempts to isolate single B infections of $N$. giraulti and $N$. longicornis through antibiotic and diapause treatment were unsuccessful, probably due to low bacterial densities of these infections in diapausing host larvae.

\section{Introgression lines}

Introgression lines were produced that harbor the cytoplasm of $N$. longicornis (infected and uninfected) in the genetic background of $N$. giraulti. [wAl, $w \mathrm{Bl}] \mathrm{G}$ carries the double-infected $N$. longicornis cytoplasm from IV7 in the $N$. giraulti genetic background of RV2R. [01]G is comprised of the uninfected cytoplasm of IV7R3-1B and the same $N$. giraulti genetic background of RV2R. These introgression lines were generated by six generations of backcrossing hybrid females to males of $N$. giraulti. This design should theoretically result in at least a $98 \%$ genome replacement, and the retaining of the cytoplasm of the parental female (infected or uninfected). Crosses with these introgression lines and pure $N$. giraulti lines that carry a $N$. giraulti cytoplasm were set up according to the methods described below.

\section{Crossing design}

All crosses were set up as single pair matings between virgin females and virgin males. Males and females were collected as pupae. Individual female and male adults were paired and observed for $10-15 \mathrm{~min}$. Only those pairings where copulation occurred were used. After $24 \mathrm{~h}$, the males were discarded and each female was provided with four hosts and a drop of honey for feeding. After $48 \mathrm{~h}$, the females were transferred to new vials and given a single host for $6 \mathrm{~h}$. Females were then discarded from each vial and the parasitized hosts were left undisturbed until adult emergence in approximately 2 weeks. Adults were scored upon death for sex and total family size. Crosses producing diapause offspring were not included in the scoring.

\section{Statistics}

We present descriptive statistics and significance values from nonparametric Mann-Whitney $U$ (MWU) tests using MINITAB 12.23. Summary data are indicated as percentages or as means \pm standard errors (s.e.) of offspring number.

\section{Results}

Results can be summarized as follows: (i) all three divergent A Wolbachia strains in the three Nasonia species constitute different incompatibility types. (ii) The codiverging strains of B Wolbachia in N. giraulti and $N$. longicornis differ in CI penetrance with the latter 
[wAI]L x [wAv]V

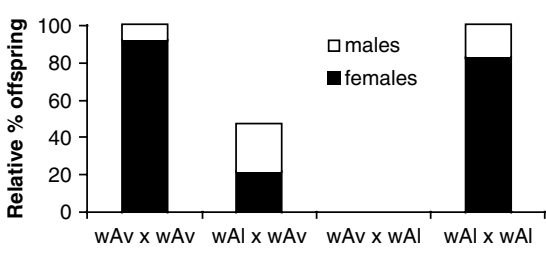

[OI]L x [0v]V

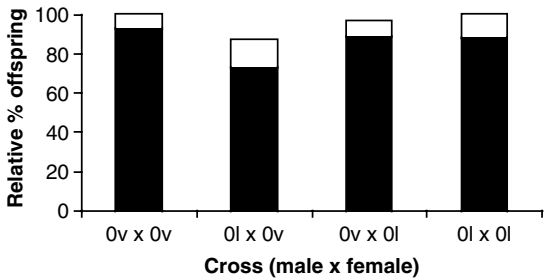

[wAI]L $x$ [wAg]G

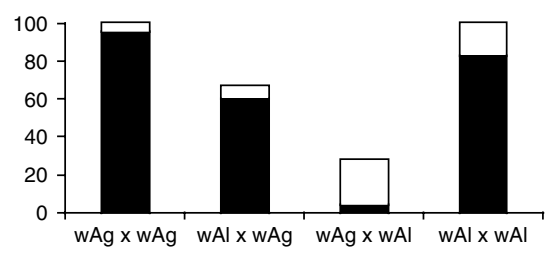

[OI]L $\times[0 \mathrm{~g}] \mathrm{G}$

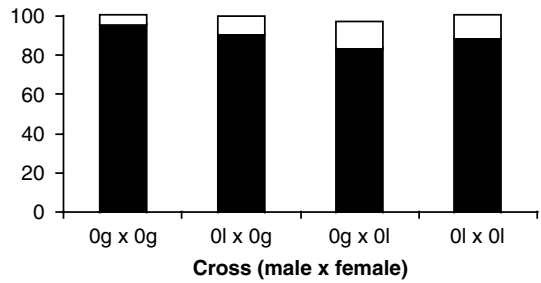

[wAv]V x [wAg]G

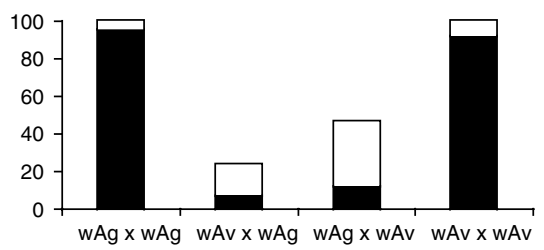

$[0 \mathrm{v}] \mathrm{V} \times[\mathrm{Og}] \mathrm{G}$

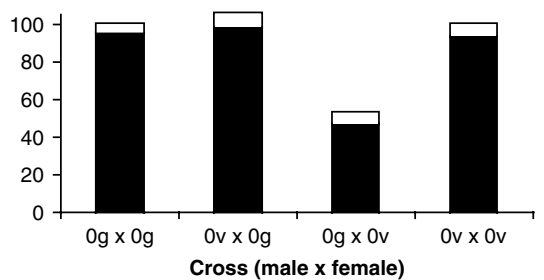

Figure 2 Bidirectional cytoplasmic incompatibility (CI) between each of the distantly related A Wolbachia strains of Nasonia. Data are represented as percent males and females based on the mean number of male and female offspring produced. Data from compatible selfcrosses are standardized so that total offspring produced equates to $100 \%$ to control for fertility differences between the three species; incompatible crosses are standardized according to the same scale for compatible crosses with the same maternal parent. Crosses are always listed as male $\times$ female.

inducing weak or no CI. (iii) The host genetic background does not influence bidirectional CI of double AB infections between the sister species $N$. giraulti and $N$. longicornis. Previous work had also showed no host genetic effects on bidirectional CI between $N$. giraulti and $N$. vitripennis (Breeuwer and Werren, 1993a). In interpreting the results below, it should be kept in mind that in compatible crosses fertilized eggs normally result in only female offspring, whereas males result from unfertilized eggs. Therefore, CI is documented by a reduction in the number of female progeny, and can be due to mortality of female embryos (which does not increase the number of male progeny) or conversion of diploid embryos into haploid males (which does increase the number of male progeny). The relative level of mortality and conversion CI can therefore be determined by comparing numbers of sons and daughters in incompatible crosses to compatible control crosses.

\section{Bidirectional $\mathrm{Cl}$ between distantly related A Wolbachia strains}

The A Wolbachia of all three species of Nasonia are not closely related, indicating independent acquisition of all three bacteria by horizontal transfer from other sources (Figure 1; Breeuwer et al., 1992; Werren et al., 1995a; van Opijnen et al., 2005). The modification and rescue components of these three A strains were tested for whether they were sufficiently different to render them bidirectionally incompatible. Experiments were done with each strain within its respective host species genetic background.

Figure 2 summarizes the results of these compatibility tests using relative percent offspring produced to standardize differences in fertility between the species. Crosses between all three A Wolbachia-infected wasps show significant decreases in diploid female production in comparison to control uninfected crosses and selfcrosses that have the same maternal parent. For example, bidirectional $\mathrm{CI}$ between the $\mathrm{A}$ infections of $N$. longicornis and $N$. vitripennis yields a $76.9 \%$ reduction in the number of daughters in the $w \mathrm{Al}$ male $\times w \mathrm{Av}$ female cross direction (mean \pm s.e.: $8.7 \pm 3.8$ daughters, $N=9$ vs $37.6 \pm 2.3$ daughters produced in the $w \mathrm{Av} \times w$ Av control self-cross, $N=27$, MWU, $P=0.0001$ ) and a $100 \%$ reduction in the reciprocal $w \mathrm{Av}$ male $\times w \mathrm{Al}$ female cross direction $(0.0 \pm 0.0$ daughters, $N=4$ vs $10.7 \pm 1.92$ control daughters, $N=10, P=0.0053)$. In a replicate experiment, we determined that the partial CI in the $w \mathrm{Al}$ male $\times w \mathrm{Av}$ female cross is repeatable and results from an incomplete $w \mathrm{Al}$ modification of the sperm rather than partial rescue. The $w \mathrm{Al}$ male $\times 0 \mathrm{v}$ female cross shows similar levels of partial CI $(4.7 \pm 1.1$ daughters, $N=17)$ when compared to the $0 \mathrm{v} \times 0 \mathrm{v}$ control self-cross $(28.5 \pm 1.6$ daughters, $N=27, P<0.0001$, data not shown). Approximately $21 \%$ of the F1 incompatibility in the $w \mathrm{Al}$ male $\times w \mathrm{Av}$ female cross may be due to interspecific F1 hybrid mortality as 01 males $\times 0 \mathrm{v}$ females gives significantly fewer daughters $(24.5 \pm 1.7, N=30)$ than that of the control $0 \mathrm{v}$ male $\times 0 \mathrm{v}$ female cross $(31.0 \pm 0.9, N=31)$ (MWU, $P=0.001)$.

Bidirectional $\mathrm{CI}$ between the $\mathrm{A}$ infections of the sister species $N$. longicornis and N. giraulti causes a $36.9 \%$ reduction in female offspring number in the $w \mathrm{Al}$ male $\times w \mathrm{Ag}$ female cross $(15.9 \pm 1.0$ daughters, $N=18$ vs $25.2 \pm 1.1$ control daughters, $N=27$, MWU, $P<0.0001$ ) and a $95.3 \%$ reduction in the reciprocal $w \mathrm{Ag}$ male $\times w \mathrm{Al}$ female cross $(0.5 \pm 1.0$ daughters, $N=6$ vs $10.7 \pm 1.92$ control daughters, $N=10$, MWU, $P=0.04$ ). In a replicate experiment, we found that this asymmetry in incompatibility strength is repeatable and again due to partial sperm modifications of the respective male infections. The incomplete modification of the sperm is apparent when comparing the number of daughters of the $w \mathrm{Al}$ male $\times 0 \mathrm{~g}$ female cross to that of the uninfected $0 \mathrm{~g}$ male $\times 0 \mathrm{~g}$ female control $(8.3 \pm 1.6, N=23$ vs $17.0 \pm 1.7$, $N=28$ ), which shows a $51.2 \%$ reduction in female offspring. Because females are uninfected in these cases, there is no rescue to be expected. Similarly, wAg male $\times 01$ female causes a $94.3 \%$ reduction in female offspring $(0.5 \pm 0.2, N=31)$ relative to that of the uninfected 01 male $\times 01$ female cross $(8.7 \pm 1.3, N=30)$. 
And finally, bidirectional CI between the A infections of $N$. vitripennis and $N$. giraulti causes a $92.5 \%$ reduction in $w \mathrm{Av}$ male $\times w \mathrm{Ag}$ female relative to the control $0 \mathrm{v}$ male $\times$ wAg female $(1.9 \pm 0.7, N=9$ vs $25.2 \pm 1.1$ control, $N=27$, MWU, $P<0.0001)$ and a $87.0 \%$ reduction in the reciprocal $w \mathrm{Ag}$ male $\times w \mathrm{Av}$ female cross vs the control $(4.9 \pm 3.0, N=7$ vs $37.6 \pm 2.3$ control, $N=27, \mathrm{MWU}$, $P=0.0001)$. Bidirectional incompatibility of these Nasonia A infections has been documented previously (Bordenstein and Werren, 1998; Werren, 1998).

In summary, all three divergent A strains from each of the Nasonia species are bidirectionally incompatible when in their own respective host genetic background. Partial incompatibility in these crosses results from incomplete A Wolbachia sperm modifications, rather than partial rescuing of that modification in the A-infected fertilized eggs.

\section{$\mathrm{Cl}$ levels of recently diverged B Wolbachia strains}

In contrast to the horizontally acquired A infections, the closely related B Wolbachia in N. giraulti and N. longicornis appear to have codiverged during divergence of their respective host species (Figure 1) (van Opijnen et al., 2005). The occurrence of such closely related Wolbachia within hosts that can be hybridized presents a rare opportunity to characterize the changes in CI that occur among recently diverged Wolbachia variants. Because single B-infected strains of these two species have proven difficult to generate, we were restricted to characterizing unidirectional CI only via crosses between doubleinfected males (for example, $w \mathrm{Ag}, w \mathrm{Bg}$ ) and single A-infected females (for example, wAg). The reason is that while the sperm modification of type A Wolbachia will be rescued in the A-infected egg, the sperm modification of type B Wolbachia will not be rescued because the B infection is absent from the egg (Mercot et al., 1995; Sinkins et al., 1995; Perrot-Minnot et al., 1996; Dobson et al., 2001).

Figure 3 summarizes the results of these compatibility tests. $w \mathrm{Bg}$ induces nearly complete $\mathrm{CI}$, but $w \mathrm{Bl}$ induces weak or no $\mathrm{CI}$ within its resident species genetic background. This finding can be seen by comparing the number of females produced in the following crosses. When $w \mathrm{Ag}, w \mathrm{Bg}$ males are crossed to $w \mathrm{Ag}$ females $(N=34)$, the number of females produced are significantly reduced from that of the control $w \mathrm{Ag}$ male $\times w \mathrm{Ag}$ female self-cross $(N=19)$ (MWU, $P<0.0001)$. These results indicate that the $w \mathrm{Bg}$ bacterium in the male has modified the sperm and the wAg-infected egg is incapable of rescuing that modification. The conclusion is further supported by comparing the $w \mathrm{Ag}, w \mathrm{Bg}$ male $\times 0 \mathrm{~g}$ female cross $(N=15)$ to the $w \mathrm{Ag}$ male $\times 0 \mathrm{~g}$ female cross $(N=24)$. Here $C I$ is observed in both cases, but when $w \mathrm{Ag}, w \mathrm{Bg}$ males are used, the $\mathrm{CI}$ is complete (that is, no female production), and when $w \mathrm{Ag}$ males are used, the CI is incomplete (that is, some females produced). Thus, double-infected males express stronger levels of $\mathrm{CI}$ than single A-infected males in $N$. giraulti. However, B-infected males do induce complete CI.

The pattern is different in N. longicornis. Genetic crosses show that $w \mathrm{Bl}$ expresses no or very weak $\mathrm{CI}$ in its own host genetic background. The $w \mathrm{Al}, w \mathrm{Bl}$ mal$\mathrm{e} \times w \mathrm{Al}$ female cross produces many daughters $(9.3 \pm 1.0$, $N=28$ ) in comparison to those of the control wAl
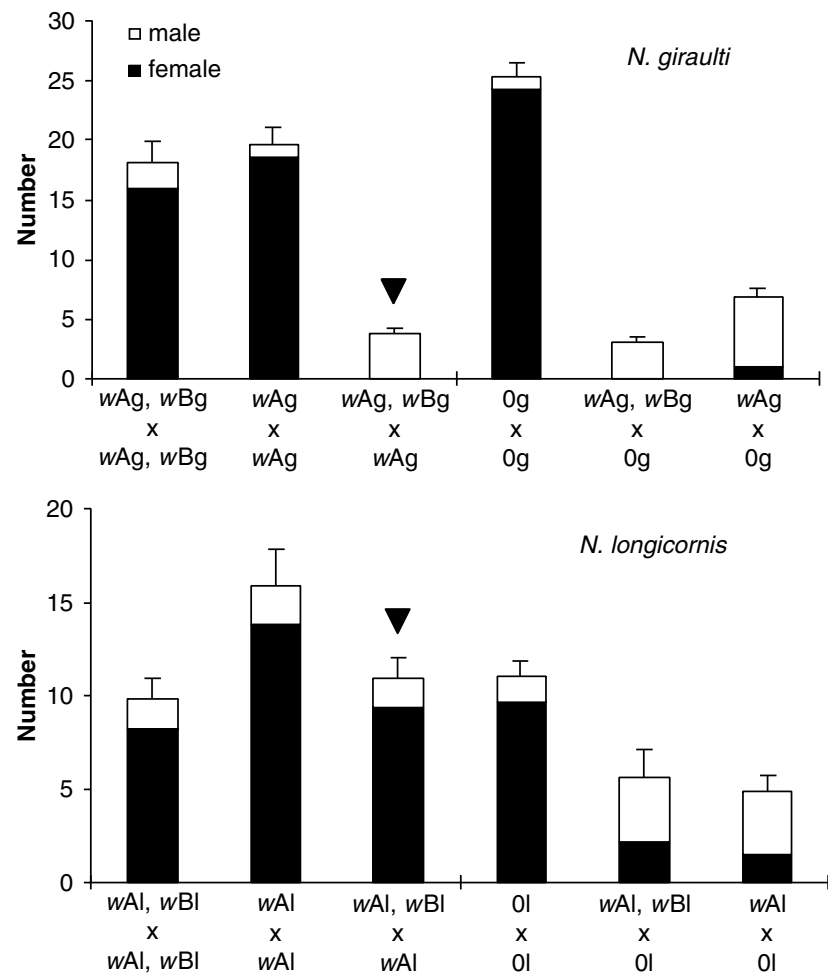

Figure 3 Unidirectional cytoplasmic incompatibility (CI) caused by the single and double infections of $N$. giraulti and $N$. longicornis. Data are shown as mean numbers of males and females produced \pm s.e. of total offspring for each cross. Cross labels denote male infection status on top line followed by crossing symbol and female infection status on the bottom line. Solid arrowheads denote the two crosses showing a significant CI level difference between the codiverging B Wolbachia of these sister wasp species.

male $\times w$ Al female self-cross $(13.9 \pm 1.7, N=7)$ (MWU, $P=0.1418$ ). The lack of significant $\mathrm{CI}$ in the former cross can be explained either by the inability of $w \mathrm{Bl}$ in a double-infected male to induce incompatibility or by $w \mathrm{Al}$ female rescue of the $w \mathrm{Bl}$ sperm modification. A comparison of the $w \mathrm{Al}, w \mathrm{Bl}$ male $\times 01$ female $(N=21)$ cross to the $w \mathrm{Al}$ male $\times 01$ female cross $(N=15)$ shows that $w \mathrm{Bl}$ does not express significant CI levels (77.1 and $84.0 \%$ reduction in the number of females, MWU, $P=0.9014)$. Taken together, these results specify that $w \mathrm{Bl}$ expresses no or weak CI. It is possible that some expression of CI could be detected with larger sample sizes in the crosses. Absence of the B Wolbachia in N. longicornis cannot account for this difference in CI level as infection status was confirmed by PCR amplification of Wolbachia 16S rDNA gene sequences before and after the experiments. $w \mathrm{Bl}$ may cause a fecundity cost in $N$. longicornis as $w \mathrm{Al}, w \mathrm{Bl}$ self-crosses $(N=17)$ produce significantly fewer daughters and total offspring than the $w A 1$ self-crosses $(N=7)$ (MWU, $P=0.03$ for both).

Taken together, results indicate that the closely related B Wolbachia of $N$. giraulti and $N$. longicornis express different levels of $\mathrm{CI}$ in their resident species genetic backgrounds. However, we were not able to determine whether they show bidirectional CI to each other, due to failures in producing single $w \mathrm{Bl}$ and $w \mathrm{Bg}$ strains. In addition, CI-induced embryonic mortality is the expected, primary CI type in N. giraulti and N. longicornis 
(Bordenstein et al., 2003), as indicated by the significant reductions in total family sizes of incompatible crosses. We estimate that the average percentage of eggs that die due to embryonic mortality from the crosses showing significant CI is $86.3 \%$ in N. giraulti and $73.7 \%$ in $N$. longicornis (see Materials and methods), respectively.

\section{Absence of host genetic influences on}

\section{$N$. giraulti - N. longicornis bidirectional $\mathrm{Cl}$}

Bordenstein et al. (2001) previously reported bidirectional CI between the double infections of these two sister species in their normal genetic background. Doubleinfected individuals were used from each species and incompatibility levels were complete in one cross direction, and incomplete in the other, reflecting the typically incomplete CI of infected N. longicornis males. To examine whether the host genome exerts any influence over bidirectional CI and the variation in levels of $\mathrm{CI}$, the $w \mathrm{Al}-$, wBl-infected $N$. longicornis cytoplasm was introgressed by backcrossing it for six generations into a $N$. giraulti genetic background. This line, denoted $[w \mathrm{Al}$, $w \mathrm{Bl}$ G as well as its uninfected counterpart [01]G, was used to retest CI against the pure $N$. giraulti lines, $[w \mathrm{Ag}$, $w \mathrm{Bg}] \mathrm{G}$ and $[0 \mathrm{~g}] \mathrm{G}$. The experimental design slightly differed from the above experiments in that single females were allowed to oviposit into two hosts for life. Therefore, family sizes are larger than those reported above.

As seen in Figure 4, bidirectional CI between these double infections is still expressed even when the $N$. longicornis Wolbachia are in a $N$. giraulti genome. Self-crosses and crosses with uninfected individuals are fully compatible and yield normal female-biased sex ratios. In incompatible crosses, the number of females is reduced by $88.4 \%$ in the $[w \mathrm{Al}, w \mathrm{Bl}] \mathrm{G}$ male $\times[w \mathrm{Ag}$, $w \mathrm{Bg}$ G female cross $(N=23)$ (MWU, $P<0.0001)$ and $99.4 \%$ in the reciprocal $[w \mathrm{Ag}, w \mathrm{Bg}] \mathrm{G}$ male $\times[w \mathrm{Al}, w \mathrm{Bl}] \mathrm{G}$ female cross $(N=25)$ (MWU, $P<0.001)$. CI levels are strong and are similar to levels from crosses showing bidirectional CI in non-introgression lines (Bordenstein et al., 2001). Therefore, the N. giraulti host genome does not affect bidirectional CI nor the incomplete levels of CI associated with $N$. longicornis Wolbachia in this introgression line. Previous work had found no host genetic effects on bidirectional CI of double $\mathrm{AB}$ infections in the species pair $N$. giraulti and $N$. vitripennis (Breeuwer and Werren, 1993a).

\section{Discussion}

The study set out to answer three questions related to $\mathrm{CI}$ in Nasonia: (i) Do the distantly related A Wolbachia of each species constitute distinct incompatibility types? (ii) Do the closely related B Wolbachia of $N$. giraulti and $N$. longicornis differ in CI? and (iii) Does the host genome influence interspecific, bidirectional incompatibility between double infections of $N$. giraulti and $N$. longicornis?

Experiments demonstrated that all three speciesspecific A infections in the Nasonia genus are bidirectionally incompatible and constitute at least three different incompatibility types. Phylogenetic relationships of these strains, based on several Wolbachia gene sequences (Werren et al., 1995a; Werren and Bartos, 2001; van Opijnen et al., 2005; Casiraghi et al., 2005; Baldo et al.,
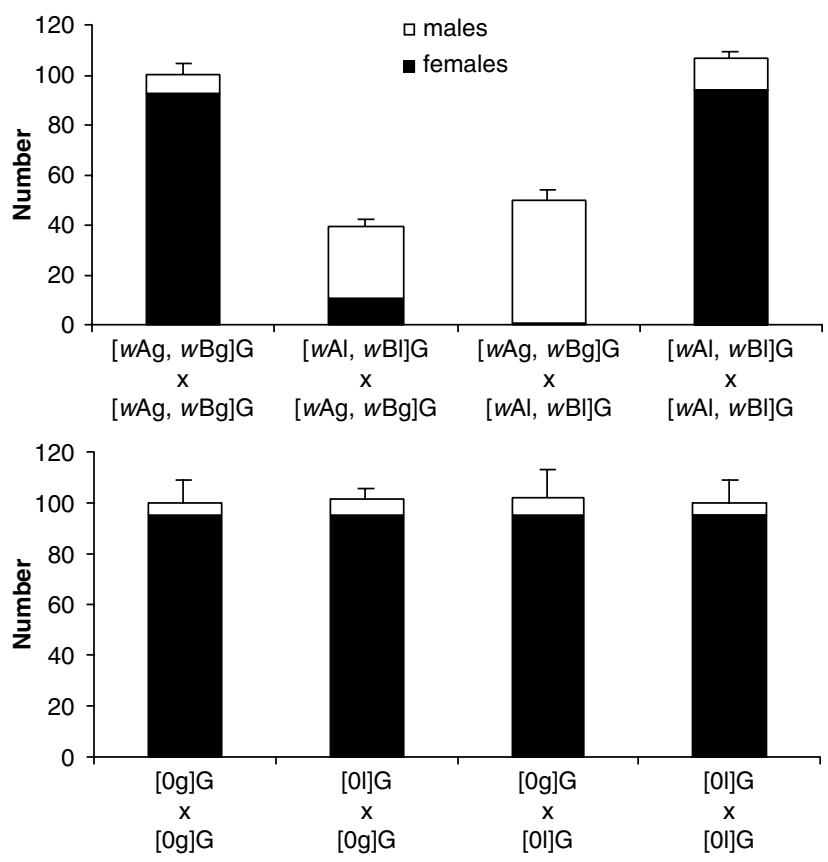

Figure 4 Bidirectional incompatibility between the double infections of $N$. longicornis and $N$. giraulti in a common $N$. giraulti genetic background. Data are shown as mean numbers of males and females produced + s.e. of total offspring for each cross. Cross labels denote male infection status on top line followed by crossing symbol and female infection status on the bottom line. Reciprocal and self-cross of infected individuals are shown above those of uninfected individuals.

2006), indicate that they are genetically divergent and were acquired in separate horizontal transfer events into the Nasonia from other unknown insects (Figure 1). Our finding of bidirectional incompatibility is consistent with this hypothesis, because there has been ample time for the modification-rescue systems of these strains to have diverged and become incompatible. Independent acquisition via lateral transfer events tends to be the predominant mechanism for how different incompatibility types arise in a host system (Bordenstein, 2003). Although little is known about the average rate of horizontal transfer for Wolbachia, it is apparent that horizontal transfer events into Nasonia can sometimes occur frequently - indeed, two separate acquisitions (two A Wolbachia) in N. giraulti and N. longicornis have happened in the last $0.2 \mathrm{Mya}$ based on their estimated divergence time (Campbell et al., 1993) (Figure 1).

An alternative mechanism for the origin of different incompatibility types is in situ evolution. That is, new incompatibility types could evolve within a species or in closely related species that have a Wolbachia that has codiverged with the host. Ultimately, new incompatibility types must arise from ancestral incompatibility types to account for the variation in CI observed among Wolbachia that have entered species by horizontal transfer. However, the process of new incompatibility type evolution is not well understood, due to a lack of knowledge about the genetic basis of CI. Charlat et al. (2001) developed a two-step model for the in situ evolution of new incompatibility types. They assume that the modification and rescue components of CI are governed by separate genes. The first step of the model 
involves the neutral spread of a mutation creating a new modification type. When this mutation drifts to an appreciable frequency, a second mutation that can rescue this new modification type will be selected for and cause the deterministic spread of this new incompatibility type under a broad set of conditions.

Models of incompatibility type evolution are difficult to test because there are very few natural examples thus far of incipient evolution of new incompatibility types within a species or between sister species. This is in part based on a limited spectrum of fastly evolving genetic markers to infer strain relationships, although transposon and phage genes may prove useful in the future (Sanogo and Dobson, 2004; Duron et al., 2005, 2006). Most studies showing bidirectional CI within their natural host species are based on Wolbachia strains that are distantly related (O'Neill and Karr, 1990; Clancy and Hoffmann, 1996; Perrot-Minnot et al., 1996; Bordenstein and Werren, 1998; James and Ballard, 2000; Dedeine et al., 2004; Mouton et al., 2005) with only few exceptions (Laven, 1957; Sinkins et al., 2005). The reason then for the paucity of data regarding these phenomena is likely a simple one. There are currently few described cases of sister CI-Wolbachia strains existing in the same or sister host species. Microinjection experiments may circumvent this problem because closely related Wolbachia that are harbored in distantly related species can be moved into a common host background (Charlat et al., 2004) and then tested for bidirectional CI. However, results produced from this approach must be interpreted with caution as host-Wolbachia interactions in the novel host may lead to confounding effects on incompatibility relationships. Nevertheless, some experiments that moved relatively closely related/identical Wolbachia of $D$. simulans and $D$. sechellia into a common $D$. simulans genetic background did not find significant differences in CI phenotype (Charlat et al., 2002).

The B Wolbachia strains of N. giraulti and N. longicornis constitute one of the best cases for natural codivergence of host and Wolbachia in insects. Sequence relationships of multiple Wolbachia genes from these B strains parallel the phylogenetic relationship of the two host species, $N$. giraulti and $N$. longicornis, which shared a direct common ancestor $\sim 0.2$ Mya (Campbell et al., 1993; Werren et al., 1995a; Werren and Bartos, 2001; van Opijnen et al., 2005; Choudhury et al., unpublished). However, a practical problem remains to determine whether they show bidirectional CI. Each strain must be isolated as a single infection from the typically double-infected individuals of each species. Crosses between them can then be performed to test for bidirectional $\mathrm{CI}$, as done with the single A infections in this study. Attempts to segregate out these single B infections, however, have only been successful in N. vitripennis (Perrot-Minnot et al., 1996). Both a prolonged diapause treatment (method described in Perrot-Minnot et al., 1996) and a low-dose antibiotic treatment have failed to segregate out the single $B$ infections of $N$. giraulti and N. longicornis (Bordenstein and Werren, unpublished). Only the A infections and cured individuals have been isolated in these sister species. A likely explanation for this outcome is a higher density of A than B Wolbachia in these species.

We therefore cannot yet determine whether bidirectional CI occurs between these two related B infections. $\mathrm{CI}$ between the distantly related B strains of $N$. vitripennis and N. giraulti has been shown (Bordenstein and Werren, 1998; Werren, 1998), suggesting that at least these B strains represent two distinct incompatibility types. In addition, despite the inability to segregate out the sister B Wolbachia strains, we can still test for differences in the expression of unidirectional CI for each of the $B$ infections by mating a double $\mathrm{AB}$-infected male to a single A-infected female. By doing so, we 'expose' the CI associated with the $B$ infection, because the A sperm modification of the double-infected individual will be rescued in the egg, but the $B$ infection will not.

The experiments reported here showed a dramatic difference in $\mathrm{CI}$ level between these two closely related B infections, $w \mathrm{Bl}$ and $w \mathrm{Bg}$ (Figure 3 ). The N. giraulti $\mathrm{B}$ strain expressed complete CI (that is, no daughters are produced), whereas the $N$. longicornis B strain expressed nearly no CI. An important question then is why is there such a drastic difference in CI level between $w \mathrm{Bl}$ and $w \mathrm{Bg}$ ? There are at least two possible explanations (a) the wBl Wolbachia lost the capability of inducing modification or (b) host genetic effects suppress modification in $w \mathrm{Bl}$. We have not yet resolved these alternatives.

$\mathrm{CI}$ level variation may be due to genetic changes in the $w \mathrm{Bl}$ and $w \mathrm{Bg}$ sister strains of Wolbachia, and represent early steps of evolutionary divergence in CI. Several models have pointed out that once a Wolbachia strain becomes fixed in a species, there is no direct selection to maintain modification function in males because Wolbachia in males are not transmitted to future generations (Prout, 1994; Turelli, 1994; Hurst and McVean, 1996). Degradation in modification function is therefore expected, either by mutation accumulation or by selection against modification because of negative pleiotropic effects in infected females. Under this scenario, the ancestral B Wolbachia of N. giraulti and N. longicornis is hypothesized to be a strong CI inducer that lost its ability to induce complete CI in N. longicornis after divergence. This conclusion is supported by the fact that the B infections in $N$. giraulti and $N$. longicornis are at near fixation (Bordenstein et al., 2001).

The B Wolbachia of $N$. longicornis may simply occur at lower densities and therefore cause lower CI levels. Studies in several systems, including Nasonia, show bacterial density effects on CI level (Boyle et al., 1993; Breeuwer and Werren, 1993b; Clancy and Hoffmann, 1998; Noda et al., 2001). Recently, the bacterial density effects in Nasonia vitripennis were further shown to be inversely associated with bacteriophage WO-B densities, suggesting that rates of lytic phage development may sometimes underlie the regulation of Wolbachia densities in arthropods (Bordenstein et al., 2006). If bacterial density is involved, it could be that the natural infection level of $w \mathrm{Bl}$ has fallen below the threshold for induction of CI, or that this has occurred in the particular laboratory strain used, possibly subsequent to its introduction into the laboratory.

Host genetic influences may also lead to differences in bacterial densities. Host-Wolbachia genetic interactions are well known to moderate CI levels in diverse host systems (Boyle et al., 1993; Bordenstein and Werren, 1998; Werren, 1998; McGraw et al., 2001) and may do so through several routes, including effects on processing of the sperm modification, bacterial densities and tissue tropism (Poinsot et al., 1998; Dobson et al., 1999; McGraw et al., 2001; Clark et al., 2002). Indeed, natural selection is 
expected to act upon the host genome of males to inhibit the modification action of Wolbachia, because their resulting sperm would then be compatible with uninfected eggs (Koehncke et al., unpublished). Thus, male host effects on CI are expected to evolve at the host level as well. For example, evidence indicates that Wolbachia are largely excluded from the developing sperm cysts in D. melanogaster (McGraw et al., 2001; Clark et al., 2002), probably explaining the low level of modification in this species. If such host-induced tissue tropism is responsible for the apparent absence of CI induction by $w \mathrm{Bl}$, then we must assume that it is specific to that strain, because the wAl does induce CI. However, detailed cytological studies to determine the tissue specificity of different Wolbachia strains in Nasonia have yet to be done.

The key results of this study are that all three A infections of Nasonia are distinct incompatibility types and that the two closely related B Wolbachia differ in CI level when tested in their resident species backgrounds. If we assume that these two B Wolbachia are not bidirectionally incompatible, then we can say that at least a total of five different incompatible Wolbachia strains (three As and two Bs) infect Nasonia, which were all presumably acquired by horizontal transfer from foreign sources. The one case of B Wolbachia codivergence has raised several interesting questions that warrant future studies of the incipient evolution of changes in CI, most important is whether codivergence of Wolbachia more often leads to loss-of-function mutations rather than evolutionary diversification of new incompatibility types. Finally, it is apparent from these studies that the interspecific postmating isolation caused by double infections of CI-Wolbachia in each Nasonia species (Breeuwer and Werren, 1990; Bordenstein et al., 2001) is reinforced by each single infection comprising its own incompatibility type, with the exception of $w \mathrm{Bl}$. Therefore, if stochastic segregation occurs in natural populations leading to the loss of Wolbachia infections, both A and B strains would have to be lost to fully restore interspecific postmating compatibility in Nasonia hybridizations.

\section{Acknowledgements}

We thank SA Biber, AB Lazarus, DB Mark Welch, ML Marshall, RR Choudhury, MB Saffo, JJ Wernegreen, and three anonymous reviewers for comments on this paper. This work was supported by grant EF-0328363 and DEB9981634 from the National Science Foundation to JHW and an Ernst Caspari Research Fellowship to SRB while he was at the University of Rochester. SRB acknowledges support from the NASA Astrobiology Institute (NNA04CC04A).

\section{References}

Baldo L, Dunning Hotopp JC, Jolley K, Bordenstein SR, Biber SA, Choudhury RR et al. (2006). Multilocus sequence typing system for the endosymbiont Wolbachia pipientis. Appl Environ Microbiol 72: 7098-7110.

Bordenstein SR (2003). Symbiosis and the origin of species. In: Bourtzis K, Miller T (eds). Insect Symbionts. CRC Press: New York. pp 283-304.

Bordenstein SR, Marshall ML, Fry AJ, Kim U, Wernegreen JJ (2006). The tripartite associations of bacteriophage, Wolbachia, and arthropods. PLoS Pathog 2: e43.
Bordenstein SR, O'Hara FP, Werren JH (2001). Wolbachiainduced incompatibility precedes other hybrid incompatibilities in Nasonia. Nature 409: 707-710.

Bordenstein SR, Rosengaus RB (2005). Discovery of a novel Wolbachia supergroup in Isoptera. Curr Microbiol 51: 393-398.

Bordenstein SR, Uy JJ, Werren JH (2003). Host genotype determines cytoplasmic incompatibility type in Nasonia. Genetics 164: 223-233.

Bordenstein SR, Werren JH (1998). Effects of A and B Wolbachia and host genotype on interspecies cytoplasmic incompatibility in Nasonia. Genetics 148: 1833-1844.

Boyle L, O'Neill SL, Robertson HM, Karr TL (1993). Inter- and intraspecific horizontal transfer of Wolbachia in Drosophila. Science 260: 1796-1799.

Breeuwer JAJ, Stouthamer R, Barns SM, Pelletier DA, Weisburg WG, Werren JH (1992). Phylogeny of cytoplasmic incompatibility micro-organisms in the parasitoid wasp genus Nasonia (Hymenoptera: Pteromalidae) based on $16 \mathrm{~S}$ ribosomal DNA sequences. Insect Mol Biol 1: 25-36.

Breeuwer JAJ, Werren JH (1990). Microorganism associated with chromosome destruction and reproductive isolation between two insect species. Nature 346: 558-560.

Breeuwer JAJ, Werren JH (1993a). The effect of genotype on cytoplasmic incompatibility between two species of Nasonia. Heredity 70: 428-436.

Breeuwer JAJ, Werren JH (1993b). Cytoplasmic incompatibility and bacterial density in Nasonia vitripennis. Genetics 135: 565-574.

Callaini G, Dallai R, Riparbelli MG (1997). Wolbachia-induced delay of paternal chromatin condensation does not prevent maternal chromosomes from entering anaphase in incompatible crosses of Drosophila simulans. J Cell Sci 110: 271-280.

Campbell BC, Steffen-Campbell JD, Werren JH (1993). Phylogeny of the Nasonia species complex (Hymenoptera: Pteromalidae) inferred from an internal transcribed spacer (ITS2) and 28S rDNA sequences. Insect Mol Biol 2: 225-237.

Casiraghi M, Bordenstein SR, Baldo L, Lo N, Beninati T, Wernegreen JJ et al. (2005). Phylogeny of Wolbachia pipientis based on gltA, groEL and ftsZ gene sequences: clustering of arthropod and nematode symbionts in the F supergroup, and evidence for futher diversity in the Wolbachia tree. Microbiology 151: 4015-4022.

Caspari E, Watson GS (1959). On the evolutionary importance of cytoplasmic sterility in mosquitoes. Evolution 13: 568-570.

Charlat S, Calmet C, Mercot H (2001). On the mod resc model and the evolution of Wolbachia compatibility types. Genetics 159: 1415-1422.

Charlat S, Kirgianaki A, Bourtzis K, Mercot H (2002). Evolution of Wolbachia-induced cytoplasmic incompatibility in Drosophila simulans and D. echellia. Evolution 56: 1735-1742.

Charlat S, Riegler M, Baures I, Poinsot D, Stauffer C, Mercot H (2004). Incipient evolution of Wolbachia compatibility types. Evolution 58: 1901-1908.

Clancy DJ, Hoffmann AA (1996). Cytoplasmic incompatibility in Drosophila simulans: evolving complexity. Trends Ecol Evol 11: 145-146.

Clancy DJ, Hoffmann AA (1998). Environmental effects on cytoplasmic incompatibility and bacterial load in Wolbachiainfected Drosophila simulans. Entomol Exp Appl 86: 13-24.

Clark ME, Veneti Z, Bourtzis K, Karr TL (2002). The distribution and proliferation of the intracellular bacteria Wolbachia during spermatogenesis in Drosophila. Mech Dev 111: 3-15.

Dedeine F, Vavre F, Shoemaker DD, Bouletreau M (2004). Intraindividual coexistence of a Wolbachia strain required for host oogenesis with two strains inducing cytoplasmic incompatibility in the wasp Asobara tabida. Evolution 58: 2167-2174

Dobson SL, Bourtzis K, Braig HR, Jones BF, Zhou W, Rousset F, O'Neill SL (1999). Wolbachia infections are distributed throughout insect somatic and germ line tissues. Insect Biochem Mol Biol 29: 153-160. 
Dobson SL, Marsland EJ, Rattanadechakul W (2001). Wolbachiainduced cytoplasmic incompatibility in single-and superinfected Aedes albopictus (Diptera : Culicidae). J Med Entomol 38: 382-387.

Duron O, Fort P, Weill M (2006). Hypervariable prophage WO sequences describe an unexpected high number of Wolbachia variants in mosquito Culex pipiens. Proc $R$ Soc Lond Series $B$ 273: 495-502.

Duron O, Lagnel J, Raymond M, Bourtzis K, Fort P, Weill M (2005). Transposable element polymorphism of Wolbachia in the mosquito Culex pipiens: evidence of genetic diversity, superinfection and recombination. Mol Ecol 14: 1561-1573.

Frydman HM, Li JM, Robson DN, Wieschaus E (2006). Somatic stem cell niche tropism in Wolbachia. Nature 441: 509-512.

Heath BD, Butcher RD, Whitfield WG, Hubbard SF (1999). Horizontal transfer of Wolbachia between phylogenetically distant species by a naturally occurring mechanism. Curr Biol 9: 313-316.

Huigens ME, de Almedia RP, Boons PA, Luck RF, Stouthamer R (2004). Natural interspecific and intraspecific horizontal transfer of parthenogenesis-inducing Wolbachia in Trichogramma wasps. Proc $R$ Soc Lond Series B 271: 509-515.

Hurst LD, McVean LT (1996). Clade selection, reversible evolution and the persistence of selfish elements: the evolutionary dynamics of cytoplasmic incompatibility. Proc $R$ Soc Lond Series B 263: 97-104.

Jaenike J, Dyeter KA, Cornish C, Minhas MS (2006). Asymmetrical reinforcement and Wolbachia infection in Drosophila. PLoS Biol 4: e325.

James AC, Ballard JWO (2000). Expression of cytoplasmic incompatibility in Drosophila simulans and its impact on infection frequencies and distribution of Wolbachia pipientis. Evolution 54: 1661-1672.

Jeyaprakash A, Hoy MA (2000). Long PCR inproves Wolbachia DNA amplification: wsp sequences found in $76 \%$ of sixtythree arthropod species. Insect Mol Biol 9: 393-405.

Jiggins FM, Hurst GDD, Majerus MEN (2000). Sex-ratiodistorting Wolbachia causes sex-role reversal in its butterfly host. Proc R Soc Lond Series B 267: 69-73.

Koukou K, Pavlikaki H, Kilias G, Werren JH, Bourtzis K, Alahiotis SN (2006). Influence of antibiotic treatment and Wolbachia on curing on sexual isolation among Drosophila melanogaster cage populations. Evolution 60: 87-96.

Laven H (1957). Vererbung durch Kernegene und das Problem der ausserkaryotischesn Vererbung bei Culex pipiens. I. Ausserkaryotisches Vererbung. Zeitschrift fur Vererbungslehre 88: 478-516.

Lo N, Casiraghi M, Salati E, Bazzocchi C, Bandi C (2002). How many wolbachia supergroups exist? Mol Biol Evol 19: 341-346.

McGraw EA, Merritt DJ, Droller JN, O'Neil SL (2001). Wolbachia-mediated sperm modification is dependent on the host genotype in Drosophila. Proc $R$ Soc Lond Series $B$ 268: 2565-2570.

Mercot H, Llorente B, Jacques M, Atlan A, Montchamp-Moreau C (1995). Variability within the Seychelles cytoplasmic incompatibility system in Drosophila simulans. Genetics 141 1015-1023.

Montchamp-Moreau C, Ferveur JF, Jacques M (1991). Geographic distribution and inheritance of 3 cytoplasmic incompatibility types in Drosophila simulans. Genetics 129: 399-407.

Mouton L, Henri H, Boutletreau M, Vavre F (2005). Multiple infections and diversity of cytoplasmic incompatibility in a haplodiploid species. Heredity 94: 187-192.

Noda H, Koizumi Y, Zhang Q, Deng K (2001). Infection density of Wolbachia and incompatibility level in two planthopper species Laodelphax striatellus and Sogatella furcifera. Insect Biochem Mol Biol 31: 727-737.

O'Neill SL, Karr TL (1990). Bidirectional incompatibility between conspecific populations of Drosophila simulans. Nature 348: 178-180.
Perrot-Minnot MJ, Guo LR, Werren JH (1996). Single and double infections with Wolbachia in the parasitic wasp Nasonia vitripennis: effects on compatibility. Genetics 143: 961-972.

Poinsot D, Bourtzis K, Markakis G, Savakis C, Mercot H (1998). Wolbachia transfer from Drosophila melanogaster into $D$. simulans: host effect and cytoplasmic incompatibility relationships. Genetics 150: 227-237.

Prout T (1994). Some evolutionary possibilities for a microbe that causes incompatibility in its host. Evolution 48: 909-911.

Reed KM, Werren JH (1995). Induction of paternal genome loss by the paternal sex ratio chromosome and cytoplasmic incompatibility bacteria (Wolbachia): a comparative study of early embryonic events. Mol Reprod Dev 40: 408-418.

Rigaud T, Juchault P, Mocquard JP (1997). The evolution of sex determination in isopod crustaceans. Bioessays 19: 409-416.

Rigaud T, Pennings PS, Juchault P (2001). Wolbachia bacteria effects after experimental interspecific transfers in terrestrial isopods. J Invertebr Pathol 77: 251-257.

Rowley SM, Raven RJ, McGraw EA (2004). Wolbachia pipientis in Australian spiders. Curr Microbiol 49: 208-214.

Sanogo YO, Dobson SL (2004). Molecular discrimination of Wolbachia in the Culex pipiens complex: evidence for variable bacteriophage hyperparasitism. Insect Mol Biol 13: 365-369.

Sinkins SP, Braig HR, O'Neill SL (1995). Wolbachia superinfections and the expression of cytoplasmic incompatibility. Proc R Soc Lond Series B 261: 325-330.

Sinkins SP, Walker T, Lynd AR, Steven AR, Makepeace BL, Godfray HCJ et al. (2005). Wolbachia variability and host effects on crossing type in Culex mosquitoes. Nature 436: 257-260.

Sintupachee S, Milne JR, Poonchaisri S, Baimai V, Kittayapong P (2006). Closely related Wolbachia strains within the pumpkin arthropod community and the potential for horizontal transmission via the plant. Microb Ecol 51: 294-301.

Stevens L, Giordano R, Fialho RF (2001). Male-killing, nematode infections, bacteriophage infection, and virulence of cytoplasmic bacteria in the genus Wolbachia. Annu Rev Ecol Syst 32: 519-545.

Stouthamer R, Breeuwer JAJ, Hurst GDD (1999). Wolbachia pipientis: microbial manipulator of arthropod reproduction. Annu Rev Microbiol 53: 71-102.

Telschow A, Hammerstein P, Werren JH (2002). The effect of Wolbachia on genetic divergence between populations: models with two way migration. Am Nat 160: S54-S66.

Telschow A, Hammerstein P, Werren JH (2005a). Wolbachia, reinforcement and speciation. Evolution 59: 1607-1619.

Telschow A, Yamamura N, Werren JH (2005b). Bidirectional cytoplasmic incompatibility and the stable coexistence of two Wolbachia strains in parapatric host populations. J Theor Biol 235: 265-274.

Tram U, Fredrick K, Werren JH, Sullivan W (2006). Paternal chromosome segregations during the first mitotic division determines Wolbachia-induced cytoplasmic incompatibility phenotype. J Cell Sci 119: 3655-3663.

Tram U, Sullivan W (2002). Role of delayed nuclear envelope breakdown and mitosis in Wolbachia-induced cytoplasmic incompatibility. Science 296: 1124-1126.

Turelli M 1994. Evolution of incompatibility-inducing microbes and their hosts. Evolution 48: 1500-1513.

Turelli M, Hoffmann AA (1991). Rapid spread of an inherited incompatibility factor in California Drosophila. Nature 353: 440-442.

van Opijnen T, Baudry E, Baldo L, Bartos J, Werren JH (2005). Genetic variability in the three genomes of Nasonia: nuclear, mitochondrial, and Wolbachia. Insect Mol Biol 14: 653-663.

Werren JH (1997). Biology of Wolbachia. Annu Rev Entomol 42: 587-609.

Werren JH (1998). Wolbachia and speciation. In: Howard DJ, Berlocher SH (eds). Endless Forms: Species and Speciation. Oxford University Press: New York. pp 245-260. 
Werren JH, Bartos JD (2001). Recombination in Wolbachia. Curr Biol 11: 431-435.

Werren JH, Beukeboom LW (1998). Sex determination, sex ratios, and genetic conflict. Annu Rev Ecol Syst 29: 233-261.

Werren JH, Guo LR, Windsor DW (1995b). Distribution of Wolbachia in neotropical arthropods. Proc R Soc Lond Series B 262: 197-204.

Werren JH, Guo LR, Zhang W (1995a). Evolution and phylogeny of Wolbachia: reproductive parasites of arthropods. Proc $R$ Soc Lond Series B 261: 55-71.
Werren JH, O'Neill SL (1997). The evolution of heritable symbionts. In: O'Neill SL, Hoffmann AA, Werren JH (eds). Influential Passengers: Inherited Microorganisms and Arthropod Reproduction. Oxford University Press: Oxford. pp 1-41.

Werren JH, Windsor DM (2000). Wolbachia infection frequencies in insects: evidence of a global equilibrium? Proc $R$ Soc Lond Series B 267: 1277-1285.

Whiting AR (1967). The biology of the parasitic wasp Mormoniella vitripennis. Q Rev Biol 42: 333-406. 